\title{
Modeling the effects of vaccination on rotavirus infection
}

\author{
Onyango Lawrence Omondi ${ }^{1,2 *}$, Chuncheng Wang ${ }^{1}$, Xiaoping Xue ${ }^{1}$ and Owuor George Lawi ${ }^{3}$
}

"Correspondence: lawionyi@gmail.com

'Department of Mathematics, Harbin Institute of Technology, Harbin, 150001, P.R. China 2 Department of Mathematics, Egerton University, P.O. Box 536, Egerton, 20115, Kenya

Full list of author information is available at the end of the article

\section{Springer}

\begin{abstract}
In this article, a mathematical model of a rotavirus infection incorporating vaccination has been developed and comprehensively analyzed. The basic reproduction number, $R_{v}$, has been established. Proof of existence of a positive endemic equilibrium has been derived. Through the analysis and simulation, it has been shown that both disease-free and endemic equilibria are globally asymptotically stable provided that $R_{v}<1$ and $R_{v}>1$, respectively. Real data has been fitted to the model, showing that it can be used to predict the nature of a rotavirus infection in a population. The results of both the analysis and the simulation show that vaccination is a very effective way of controlling rotavirus infection.
\end{abstract}

Keywords: rotavirus; vaccination; global asymptotic stability; positive endemic equilibrium; basic reproduction number

\section{Introduction}

Rotavirus is a pathogen of the gastrointestinal tract that causes acute gastroenteritis and diarrhea in infants and young children of below five years of age worldwide [1,2]. In fact, 95\% of children worldwide are infected by the time they reach age 5 with peak incidence occurring between ages of four months and 36 months [3]. It gets its name from the fact that, under a microscope, the virus resembles a wheel. Severe rotavirus infections occur most commonly in infants and children below six to 24 months of age. Its symptoms, which may last for eight days, include; fever, nausea, vomiting, abdominal cramps, and frequent watery diarrhea [4-6]. There are seven species of rotavirus, referred to as $A, B$, C, D, E, F, and G. Humans are primarily infected by species A, B, and C, most commonly by $\mathrm{A}$. The diagnosis of a rotavirus infection is commonly made clinically, although a rapid antigen stool test is available. Rotavirus is primarily transmitted by the faecal-oral route, via contact with contaminated hands, surfaces and objects, and possibly by the respiratory route [7-9]. For further information on diseases with multiple routes of infection, see [10, 11]. The incubation period is about two days [12, 13]. Reinfection does occur; however, with each infection, immunity develops, so subsequent infections are less severe [14]. Indeed, it has been observed in [15] that children who experienced two natural rotavirus infection had complete protection against moderate-to-severe diarrhea compared to children without a previous infection. It has also been established that both symptomatic and asymptomatic infections confer a similar degree of protection [15].

( 2015 Omondi et al. This article is distributed under the terms of the Creative Commons Attribution 4.0 International License (http://creativecommons.org/licenses/by/4.0/), which permits unrestricted use, distribution, and reproduction in any medium, provided you give appropriate credit to the original author(s) and the source, provide a link to the Creative Commons license, and indicate if changes were made. 
It is estimated that about $90 \%$ of children worldwide will have experienced a rotavirus infection by age five [16] with an annual mortality in excess of 600,000 among children $[17,18]$. Rotavirus-related hospitalizations can account for as many as $2.5 \%$ of all hospitalizations of children. Some review analyses show that rotavirus accounted for $6 \%$ of diarrhea episodes and $20 \%$ of deaths caused by diarrhea in children less than five years of age in developing countries [19]. In a study carried out in Ghana, it was observed that $11.8 \%$ of the 243 children examined were co-infected with plasmodium falciparum and enteropathogens, where rotavirus was also found to be one of the enteropathogens common in more than half of the patients [20]. In Kenya, rotavirus causes more than 7,500 deaths yearly. The results of a study of rotavirus infections among HIV-infected children in Nairobi, Kenya, indicate that rotavirus is an important viral etiological agent causing diarrhea in HIV - seropositive children [21].

The incidence of the rotavirus infection has been observed to be similar in both industrialized and developing countries, suggesting that adequate control measures may not be achieved by improvements in water supply, hygiene or sanitation. Consequently, development, trial, and widespread use of rotavirus vaccines are recommended to prevent both severe and fatal rotavirus disease [19]. So far, no specific cure for rotavirus infection has been discovered. Therefore disease control primarily involves preventing dehydration. Recent compelling data on the disease burden of rotavirus and the power of vaccines to prevent it in low-resource settings informed the WHO's June 2009 recommendation that rotavirus vaccination be included in every nation's immunizations program [22].

In this work, we have developed a new mathematical model for rotavirus infection that incorporates vaccination. We have analyzed it comprehensively and proved that a positive endemic equilibrium $E^{*}\left(S^{*}, V^{*}, I^{*}\right)$ exists and is globally stable. In addition, we have performed numerical simulations to confirm and demonstrate the validity of the various assumptions and conclusions made during the analysis. The paper is organized as follows. In this section, we have provided background information as regards rotavirus infection; in Section 2, we developed the model; Section 3 established a disease-free equilibrium and derived the basic reproduction number, $R_{v}$. We have also proved the existence of a positive endemic equilibrium in this section and finally proved that the disease-free equilibrium is globally asymptotically stable. In Section 4 , we proved that the endemic equilibrium is globally asymptotically stable; numerical simulation is performed in Section 5. Finally, a discussion and conclusion is presented in Section 6.

\section{Model formulation and description}

The total human population size, $N$, at any time is subdivided into classes: susceptible $S$, infectious with rotavirus $I$, vaccinated $V$ and removed $R$. Since the incubation period is very short, we assume that the probability of survival till the infectious state for the individuals exposed to rotavirus is unity and therefore exclude the exposure stage. The individuals infected with rotavirus include both symptomatic and asymptomatic cases [18]. The removed class comprises those who have been removed from the scene of infection by such means as infection-acquired immunity [15] and death. Although it is possible to have some level of immunity to rotavirus from maternal antibodies due to breastfeeding [23], we explore the effect of vaccination at birth and vaccination of susceptibles. The human population is not assumed to be constant, since birth, migration, emigration, and death occur. The recruitment into susceptible population takes place at the rate of $(1-\rho) \Lambda$, 
while recruitment into the vaccinated class occurs at the rate of $\rho \Lambda$. Susceptibles are subsequently vaccinated at the rate $\gamma$, while the vaccine efficacy wanes at the rate of $\omega$. The parameter $0<\epsilon<1$ models the expected decrease in the risk of infection as a result of vaccination. Disease mortality is assumed to take place at the rate $\tau$, while the rate of flow into the removed class is taken as $\kappa$. Let $\mu$ define the per capita natural death rate. We assume that the mass-action incidence transmission is defined by $\beta S I$ where $\beta$ is the effective contact rate for disease transmission.

From the above definitions and variables, we have the following model with nonnegative initial conditions:

$$
\begin{aligned}
& \frac{d S}{d t}=(1-\rho) \Lambda-\beta S I-\gamma S+\omega V-\mu S, \\
& \frac{d V}{d t}=\rho \Lambda+\gamma S-\epsilon \beta V I-(\omega+\mu) V, \\
& \frac{d I}{d t}=\beta S I+\epsilon \beta V I-(\tau+\kappa+\mu) I, \\
& \frac{d R}{d t}=\kappa I-\mu R .
\end{aligned}
$$

Since $N=S+V+I+R$, we have

$$
\frac{d N}{d t}=\Lambda-\mu N-\tau I
$$

From (2) we note that in the absence of infection we get $\frac{d N}{d t}=\Lambda-\mu N$, so that $N$ would approach carrying capacity $\frac{\Lambda}{\mu}$. Model (1) describes the human population and therefore it can be shown that the associated state variables are non-negative for all time $t \geq 0$ and that the solutions of the model (1) with positive initial data remain positive for all time $t \geq 0$. Thus (1) is mathematically well posed and its dynamics can be considered in a proper subset $\Omega=\left\{(S, V, I, R) \in \mathbf{R}_{+}^{4}: N \leq \frac{\Lambda}{\mu}\right\}$.

\section{Equilibrium points of the model}

The first three equations in (1) do not contain terms in $R$ and therefore we can analyze the following reduced model:

$$
\begin{aligned}
& \frac{d S}{d t}=(1-\rho) \Lambda-\beta S I-\gamma S+\omega V-\mu S, \\
& \frac{d V}{d t}=\rho \Lambda+\gamma S-\epsilon \beta V I-(\omega+\mu) V, \\
& \frac{d I}{d t}=\beta S I+\epsilon \beta V I-(\tau+\kappa+\mu) I .
\end{aligned}
$$

The equilibrium points of (3) are obtained by equating the derivatives to zero and solving for the variables. It can be shown, by using $S+V=\frac{\Lambda}{\mu}$ that the disease-free equilibrium of the model is defined by

$$
E^{0}=\left(S^{0}, V^{0}, I^{0}\right)=\left\{\frac{\mu(1-\rho) \Lambda+\omega \Lambda}{\mu(\gamma+\omega+\mu)}, \frac{(\rho \mu+\gamma) \Lambda}{\mu(\gamma+\omega+\mu)}, 0\right\} .
$$




\subsection{The basic reproduction number $R_{v}$}

The global dynamics of model (1) are highly dependent on an important epidemiological parameter called the basic reproduction number. It is defined as the average number of secondary infections an infectious individual would cause over his infectious period in an entirely susceptible population $[24,25]$. We specifically define the basic reproduction number $R_{v}$ of the model (1) as the number of secondary rotavirus infections caused by a single rotavirus infected individual in the presence of vaccination. When no such intervention is employed then the basic reproduction number is defined by $R_{0}$. When the basic reproduction number is greater than one it means that an infectious individual is causing, on average, more than one new infection and thus the disease invades and persist in the population. Using the next-generation operator approach $[25,26]$, we determine $R_{v}$ as

$$
R_{v}=\frac{\beta}{\mu(\tau+\kappa+\mu)}\left[\frac{\mu(1-\rho) \Lambda+\omega \Lambda+\epsilon \Lambda(\rho \mu+\gamma)}{(\gamma+\mu+\omega)}\right] .
$$

In the absence of vaccination, $\rho=\omega=\gamma=0$ and the basic reproduction number becomes

$$
R_{0}=\frac{\beta \Lambda}{\mu(\tau+\kappa+\mu)} .
$$

Using (5), we can express (4) as

$$
R_{\nu}=R_{0}\left[\frac{\mu(1-\rho+\rho \epsilon)+\omega+\epsilon \gamma}{(\gamma+\mu+\omega)}\right] .
$$

Since we have set $0<\epsilon<1$, it follows that $\frac{\mu(1-\rho+\rho \epsilon)+\omega+\epsilon \gamma}{(\gamma+\mu+\omega)}<1$, which implies that $R_{v}<R_{0}$. When $\epsilon=1$ or $\rho=\omega=\gamma=0, R_{v}=R_{0}$. Thus we see from the expression of $R_{v}$ that the vaccination from birth as well as vaccination of susceptibles both have a positive impact on the reduction of new infections. Note that $\epsilon=1$ implies that vaccination is not important and this should not be the case. Though we have used a next-generation approach to calculate $R_{0}$, it is worth noting that there are other methods that can be used in models where it fails. For example, the survival function, the eigenvalues of the Jacobian matrix, the existence of endemic equilibrium or the constant characteristic polynomial. All these methods have their own limitations. For further details, see [27].

We now establish the existence of an endemic equilibrium. This is done in the lemma below.

\subsection{Existence of a unique positive endemic equilibrium $E^{*}\left(S^{*}, V^{*}, I^{*}\right)$}

Lemma 1 An endemic equilibrium $E^{*}\left(S^{*}, V^{*}, I^{*}\right)$ exists provided that $R_{v}>1$.

Proof At an endemic state, equation (3) becomes

$$
\begin{aligned}
& 0=(1-\rho) \Lambda-\beta S^{*} I^{*}-\gamma S^{*}+\omega V^{*}-\mu S^{*}, \\
& 0=\rho \Lambda+\gamma S^{*}-\epsilon \beta V^{*} I^{*}-(\omega+\mu) V^{*}, \\
& 0=\beta S^{*} I^{*}+\epsilon \beta V^{*} I^{*}-(\tau+\kappa+\mu) I^{*} .
\end{aligned}
$$


Solving for $S^{*}$ from the first equation in (7), we obtain

$$
S^{*}=\frac{(1-\rho) \Lambda+\omega V^{*}}{\beta I^{*}+\gamma+\mu} \text {. }
$$

Solving for $V^{*}$ from the second equation of (7), we get

$$
V^{*}=\frac{\rho \Lambda+\gamma S^{*}}{\epsilon \beta I^{*}+\omega+\mu}
$$

Again, from the third equation of (7), we obtain

$$
S^{*}=\frac{(\tau+\kappa+\mu)-\epsilon \beta V^{*}}{\beta} .
$$

Equating (8) to (10) and solving for $V^{*}$, we get

$$
V^{*}=\frac{(\tau+\kappa+\mu)\left(\beta I^{*}+\gamma+\mu\right)-\beta(1-\rho) \Lambda}{\beta\left(\omega+\epsilon\left(\beta I^{*}+\gamma+\mu\right)\right)} .
$$

Substituting (10) into (9) and simplifying, we get

$$
V^{*}=\frac{\beta \rho \Lambda+\gamma(\tau+\kappa+\mu)}{\beta\left(\epsilon \beta I^{*}+\omega+\mu+\epsilon \gamma\right)} .
$$

To obtain the value of $I^{*}$, we equate (11) to (12) and simplify to get

$$
\begin{gathered}
\mu \rho \beta \Lambda-\epsilon \mu \rho \beta \Lambda-\gamma \epsilon \beta \Lambda+\gamma \epsilon \beta(\tau+\kappa+\mu) I^{*}+\gamma \mu(\tau+\kappa+\mu)-\epsilon \beta^{2} \Lambda I^{*} \\
+\epsilon \beta^{2}(\tau+\kappa+\mu) I^{* 2}+\epsilon \mu \beta(\tau+\kappa+\mu) I^{*}-\omega \beta \Lambda+\omega \beta(\tau+\kappa+\mu) I^{*} \\
+\mu \omega(\tau+\kappa+\mu)-\mu \beta \Lambda+\mu \beta(\tau+\kappa+\mu) I^{*}+\mu^{2}(\tau+\kappa+\mu)=0,
\end{gathered}
$$

which we express as $A I^{* 2}+B I^{*}+C=0$, where

$$
\begin{aligned}
A= & \epsilon \beta^{2}(\tau+\kappa+\mu), \\
B= & {\left[\gamma \epsilon \beta(\tau+\kappa+\mu)-\epsilon \beta^{2} \Lambda+\epsilon \mu \beta(\tau+\kappa+\mu)+\omega \beta(\tau+\kappa+\mu)+\mu \beta(\tau+\kappa+\mu)\right], } \\
C= & {[\mu \rho \beta \Lambda-\epsilon \mu \rho \beta \Lambda-\gamma \epsilon \beta \Lambda+\gamma \mu(\tau+\kappa+\mu)-\omega \beta \Lambda+\mu \omega(\tau+\kappa+\mu)} \\
& \left.-\mu \beta \Lambda+\mu^{2}(\tau+\kappa+\mu)\right] .
\end{aligned}
$$

To determine the sign of $C$, we express it as

$$
C=\mu[\beta \rho \Lambda+(\tau+\kappa+\mu)(\gamma+\omega+\mu)]-\beta \Lambda[\epsilon \mu \rho+\epsilon \gamma+\omega+\mu] .
$$

Since $R_{v}=\frac{\beta}{\mu(\tau+\kappa+\mu)}\left[\frac{\mu(1-\rho) \Lambda+\omega \Lambda+\epsilon \Lambda(\rho \mu+\gamma)}{(\gamma+\mu+\omega)}\right]>1$, it is easy to show that

$$
\beta \Lambda[\mu+\omega+\epsilon \mu \rho+\gamma \epsilon]>\mu[\beta \rho \Lambda+(\tau+\kappa+\mu)(\gamma+\omega+\mu)] .
$$

This proves that $C<0$, when $R_{v}>1$. We therefore see that (13) can only be expressed as either $A \lambda^{2}-B \lambda-C=0$ or $A \lambda^{2}+B \lambda-C=0$, and by the Descartes rule of sign changes there is only one positive root of (13); that is, $I^{*}>0[28,29]$. 


\subsection{Global stability of the disease-free equilibrium}

The global stability of the disease-equilibrium $E^{0}$ is easily proved by using a common quadratic Lyapunov function and LaSalle's invariance principle.

Theorem 2 If $R_{v} \leq 0$, then the disease-free equilibrium of (3) is globally asymptotically stable in $\Omega$.

Proof Define $L:\{(S, V, I) \in \Omega: S, V>0\} \rightarrow \mathbb{R}$ by

$$
L(S, V, I)=(\omega+\mu) I .
$$

Then, if $R_{0} \leq 0$,

$$
\begin{aligned}
L^{\prime} & =(\omega+\mu)[\beta S I+\epsilon \beta V I-(\tau+\kappa+\mu) I] \\
& =(\omega+\mu)[\beta(S+\epsilon V)-(\tau+\kappa+\mu)] I \\
& \leq(\omega+\mu)[\beta(S+V)-(\tau+\kappa+\mu)] I \\
& \leq(\omega+\mu)\left[\frac{\beta \Lambda}{\mu}-(\tau+\kappa+\mu)\right] I \\
& \leq\left(R_{0}-1\right)(\omega+\mu)(\tau+\kappa+\mu) I \\
& \leq\left(R_{v}-1\right)(\omega+\mu)(\tau+\kappa+\mu) I,
\end{aligned}
$$

since $R_{v}<R_{0}$ in $0<\epsilon<1$.

If $L^{\prime}=0$, then $I=0$ or $R_{v}=0$. Hence $L$ is a Lyapunov function on $\Omega$. Thus $I \rightarrow 0$ as $t \rightarrow \infty$. When we substitute $I=0$ in (3), we obtain $S+V \rightarrow \frac{\Lambda}{\mu}$. Therefore it follows from LaSalle's invariance principle [30-32] that every solution of (3), with initial conditions in $\Omega$, approaches $E^{0}$ as $t \rightarrow \infty$.

\section{Global stability of the endemic equilibrium of the model}

To prove the global stability of the endemic equilibrium, we use the Lyapunov direct method. We construct the Lyapunov function by using the logarithmic functions.

Theorem 3 If $R_{v}>1$ then the unique endemic equilibrium $E^{*}\left(S^{*}, V^{*}, I^{*}\right)$ of (3) is globally asymptotically stable in the interior of $\Omega$.

Proof Define $L:\{(S, V, I): \in \Omega: S, V, I>0\} \rightarrow \mathbb{R}$ by

$$
L=\left(S-S^{*}-S^{*} \ln \frac{S}{S^{*}}\right)+\left(V-V^{*}-V^{*} \ln \frac{V}{V^{*}}\right)+\left(I-I^{*}-I^{*} \ln \frac{I}{I^{*}}\right) .
$$

Then $L$ is $C^{1}$ on the interior of $\Omega, E^{*}$ is a global minimum of $L$ on $\Omega$ and $L\left(S^{*}, V^{*}, I^{*}\right)=0$. $\frac{d L}{d t}$ computed along the solutions of (3) is given by

$$
\begin{aligned}
L^{\prime}= & \left(1-\frac{S^{*}}{S}\right)[(1-\rho) \Lambda-\beta S I-\gamma S+\omega V-\mu S] \\
& +\left(1-\frac{V^{*}}{V}\right)[\rho \Lambda+\gamma S-\epsilon \beta V I-(\omega+\mu) V] \\
& +\left(1-\frac{I^{*}}{I}\right)[\beta S I+\epsilon \beta V I-(\tau+\kappa+\mu) I] .
\end{aligned}
$$


At the equilibrium point of (3), we have

$$
\begin{aligned}
& (1-\rho) \Lambda=\beta S^{*} I^{*}+\gamma S^{*}-\omega V^{*}+\mu S^{*}, \\
& \rho \Lambda=-\gamma S^{*}+\epsilon \beta V^{*} I^{*}+(\omega+\mu) V^{*} .
\end{aligned}
$$

Substituting (16) into (15), we obtain

$$
\begin{aligned}
L^{\prime}= & \left(1-\frac{S^{*}}{S}\right)\left[\beta S^{*} I^{*}+\gamma S^{*}-\omega V^{*}+\mu S^{*}-\beta S I-\gamma S+\omega V-\mu S\right] \\
& +\left(1-\frac{V^{*}}{V}\right)\left[-\gamma S^{*}+\epsilon \beta V^{*} I^{*}+(\omega+\mu) V^{*}+\gamma S-\epsilon \beta V I-(\omega+\mu) V\right] \\
& +\left(1-\frac{I^{*}}{I}\right)[\beta S I+\epsilon \beta V I-(\tau+\kappa+\mu) I] \\
= & \left(1-\frac{S^{*}}{S}\right)\left[-(\gamma+\mu)\left(S-S^{*}\right)+\beta S^{*} I^{*}-\beta S I-\omega V^{*}+\omega V\right] \\
& +\left(1-\frac{V^{*}}{V}\right)\left[-(\omega+\mu)\left(V-V^{*}\right)-\gamma S^{*}+\gamma S+\epsilon \beta V^{*} I^{*}-\epsilon \beta V I\right] \\
& +\left(1-\frac{I^{*}}{I}\right)[\beta S I+\epsilon \beta V I-(\tau+\kappa+\mu) I] .
\end{aligned}
$$

Using $(\tau+\kappa+\mu) I^{*}=\beta S^{*} I^{*}+\epsilon \beta V^{*} I^{*}$ and $0=\beta S^{*} I+\epsilon \beta V^{*} I-(\tau+\kappa+\mu) I$, we get

$$
\begin{aligned}
= & -\left(\frac{\gamma+\mu}{S}\right)\left(S-S^{*}\right)^{2}-\left(\frac{\omega+\mu}{V}\right)\left(V-V^{*}\right)^{2} \\
& +2 \beta S^{*} I^{*}-\beta S^{*} I^{*} \frac{S^{*}}{S}-\beta S I^{*}+2 \epsilon \beta V^{*} I^{*} \\
& -\epsilon \beta V^{*} I^{*} \frac{V^{*}}{V}-\epsilon \beta V I^{*}+\gamma S-\gamma S^{*}+\gamma S^{*} \frac{V^{*}}{V} \\
& -\gamma S \frac{V^{*}}{V}+\omega V-\omega V^{*}+\omega V^{*} \frac{S^{*}}{S}-\omega V \frac{S^{*}}{S} .
\end{aligned}
$$

Setting $\mu=(\omega+\gamma)$ and $\gamma=\frac{\omega V^{*}}{S^{*}}$, we get

$$
\begin{aligned}
\leq & \beta S^{*} I^{*}\left(2-\frac{S^{*}}{S}-\frac{S}{S^{*}}\right)+\epsilon \beta V^{*} I^{*}\left(2-\frac{V^{*}}{V}-\frac{V}{V^{*}}\right) \\
& -2 \gamma S+4 \gamma S^{*}-2 \gamma \frac{S^{* 2}}{S}-2 \omega V+4 \gamma S^{*}-2 \gamma S^{*} \frac{V^{*}}{V} \\
& -\gamma S^{*}+\omega V+\gamma \frac{S^{* 2}}{S}-\omega V \frac{S^{*}}{S}-\gamma S^{*}+\gamma S+\gamma S^{*} \frac{V^{*}}{V}-\gamma S \frac{V^{*}}{V} \\
\leq & \beta S^{*} I^{*}\left(2-\frac{S^{*}}{S}-\frac{S}{S^{*}}\right)+\epsilon \beta V^{*} I^{*}\left(2-\frac{V^{*}}{V}-\frac{V}{V^{*}}\right) \\
& -\gamma S+6 \gamma S^{*}-\omega V-\gamma \frac{S^{* 2}}{S}-\gamma S^{*} \frac{V^{*}}{V}-\omega S \frac{S^{*}}{S}-\gamma S \frac{V^{*}}{V} \\
\leq & \beta S^{*} I^{*}\left(2-\frac{S^{*}}{S}-\frac{S}{S^{*}}\right)+\epsilon \beta V^{*} I^{*}\left(2-\frac{V^{*}}{V}-\frac{V}{V^{*}}\right) \\
& +3 \gamma S^{*}-\gamma S-\gamma \frac{S^{* 2}}{S}-\gamma S^{*} \frac{V^{*}}{V}+3 \omega V^{*}-\omega V-\omega \frac{V^{* 2}}{V}-\omega V \frac{S^{*}}{S}
\end{aligned}
$$




$$
\begin{aligned}
\leq & \beta S^{*} I^{*}\left(2-\frac{S^{*}}{S}-\frac{S}{S^{*}}\right)+\epsilon \beta V^{*} I^{*}\left(2-\frac{V^{*}}{V}-\frac{V}{V^{*}}\right) \\
& +\gamma S^{*}\left(3-\frac{S^{*}}{S}-\frac{S}{S^{*}}-\frac{S V^{*}}{S^{*} V}\right)+\omega V^{*}\left(3-\frac{V}{V^{*}}-\frac{V^{*}}{V}-\frac{V S^{*}}{V^{*} S}\right) .
\end{aligned}
$$

Replacing $\omega V^{*}$ with $\gamma S^{*}$ as defined above, we get

$$
\begin{aligned}
\leq & \beta S^{*} I^{*}\left(2-\frac{S^{*}}{S}-\frac{S}{S^{*}}\right)+\epsilon \beta V^{*} I^{*}\left(2-\frac{V^{*}}{V}-\frac{V}{V^{*}}\right) \\
& +\gamma S^{*}\left(3-\frac{S^{*}}{S}-\frac{S}{S^{*}}-\frac{S V^{*}}{S^{*} V}\right)+\gamma S^{*}\left(3-\frac{V}{V^{*}}-\frac{V^{*}}{V}-\frac{V S^{*}}{V^{*} S}\right) \\
\leq & \beta S^{*} I^{*}\left(2-\frac{S^{*}}{S}-\frac{S}{S^{*}}\right)+\epsilon \beta V^{*} I^{*}\left(2-\frac{V^{*}}{V}-\frac{V}{V^{*}}\right) \\
& +\gamma S^{*}\left(6-\frac{S^{*}}{S}-\frac{S}{S^{*}}-\frac{V}{V^{*}}-\frac{V^{*}}{V}-\frac{V S^{*}}{V^{*} S}-\frac{S V^{*}}{S^{*} V}\right) \\
\leq & 0 .
\end{aligned}
$$

Thus, using the arithmetic-geometric mean inequality, $L^{\prime} \leq 0$ with equality if $S=S^{*}, V=$ $V^{*}$, and $I=I^{*}$. This implies that the largest compact invariant set in $\left\{(S, V, I) \in \Omega: L^{\prime}=0\right\}$ is the singleton $\left\{E^{*}\right\}$, where $E^{*}$ is the endemic equilibrium. Therefore, LaSalle's invariance principle [30-32] implies that $E^{*}$ is globally asymptotically stable in the interior of $\Omega$.

\section{Numerical simulations}

We now present the numerical simulation results that are used to illustrate global asymptotic stability of model (3). The parameter values in Table 1 are used in simulating Figures 1(a), 1(b), 2(a), 2(b), and 3(a). The data in Table 2 is used in fitting the model; see Figure 3(b).

\section{Discussion and conclusion}

From the numerical simulation results, Figure 1(a) shows that the disease-free equilibrium is globally asymptotically stable when $R_{v}=0.9692$, which is less than unity. In the figure, we also note that there are few individuals who are vaccinated compared to those who are susceptible. This may be due to the fact that vaccination is not necessary at this time since there are still no infections in the population; that is, $I=0$. Figure 1 (b) shows that the endemic equilibrium is globally asymptotically stable for $R_{v}=1.7195$. The figure also depicts an increase in the number of infected individuals at the onset of an infection before the global asymptotic stability is attained. Therefore it is highly recommended that

Table 1 Parameter values used in Figures 1, 2, and 3

\begin{tabular}{llll}
\hline Parameter & Symbol & Value & Source \\
\hline Recruitment rate of humans & $\Lambda$ & $4.109 \times 10^{3}$ people/day & {$[33]$} \\
Recruitment rate of vaccinated individuals & $\rho$ & $1.884 \times 10^{-3} \mathrm{people}_{\text {day }}$ & {$[34]$} \\
Natural death rate of humans & $\mu$ & $2.537 \times 10^{-5} \mathrm{day}^{-1}$ & {$[33]$} \\
Rotavirus-induced deaths & $\tau$ & $4.466 \times 10^{-5} \mathrm{day}^{-1}$ & {$[19]$} \\
Effective contact rate & $\beta$ & Variable $\mathrm{day}^{-1}$ & Variable \\
Rate of flow into the removed class & $\kappa$ & $9.5 \times 10^{-4} \mathrm{day}^{-1}$ & Estimated \\
Expected decrease in the risk of infection & $\epsilon$ & $1.0 \times 10^{-3} \mathrm{day}^{-1}$ & Assumed \\
Vaccine efficacy waning rate & $\omega$ & $2.778 \times 10^{-3} \mathrm{day}^{-1}$ & {$[35]$} \\
Vaccination rate & $\gamma$ & $1.884 \times 10^{-3} \mathrm{day}^{-1}$ & {$[34]$} \\
\hline
\end{tabular}




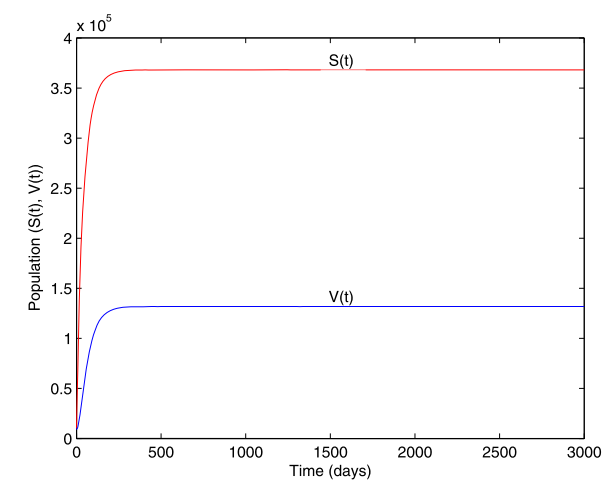

(a)

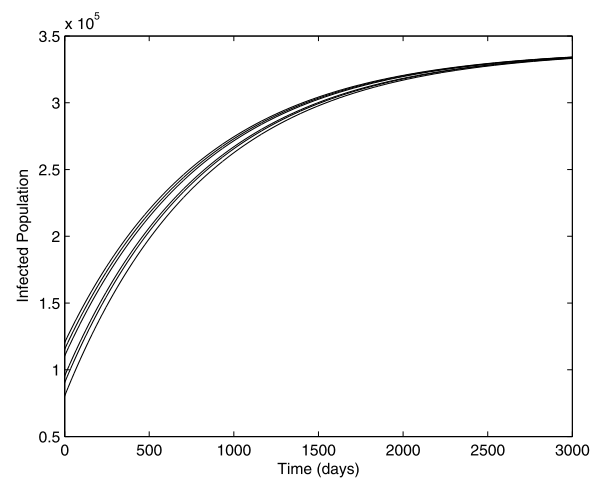

(b)

Figure 1 Simulation of model (3). Figure 1 (a) shows the global stability of the disease-free equilibrium when $R_{v}=0.9692$. Figure 1 (b) shows the global stability of the endemic equilibrium plotted at various initial values with $R_{v}=1.7195$. All other parameters are shown in Table 1 .

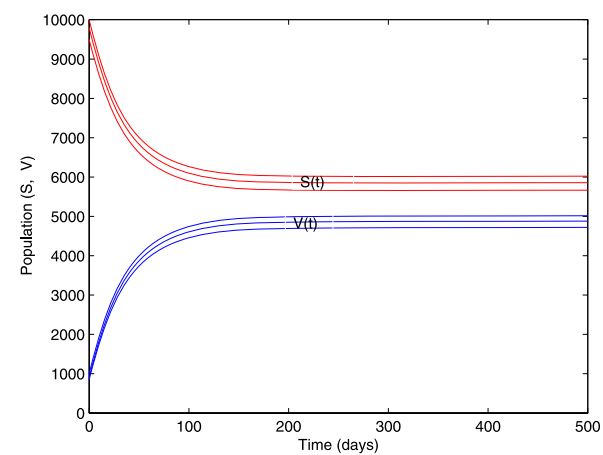

(a)

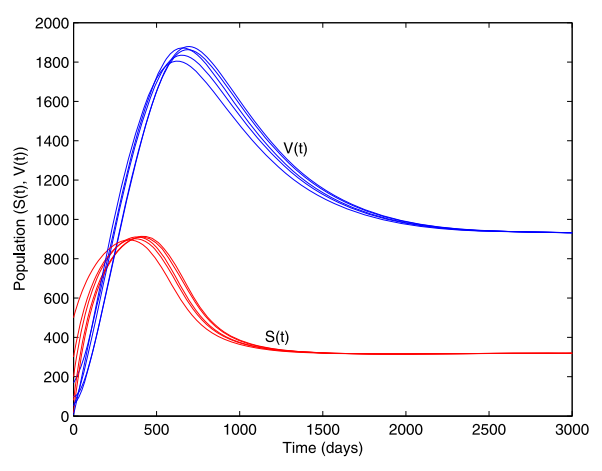

(b)

Figure 2 Figure 2 shows how varying $\epsilon$ affects both the susceptible and the vaccinated population. In Figure 2 (a) $\epsilon=0.01$ with $R_{v}=1.6135$ and in Figure $2(\mathbf{b}) \epsilon=0.001$ and $R_{v}=1.2163$. All other parameters remain as in Table 1.

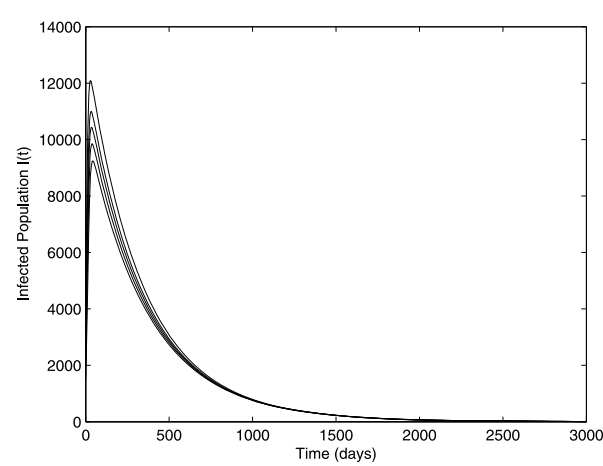

(a)

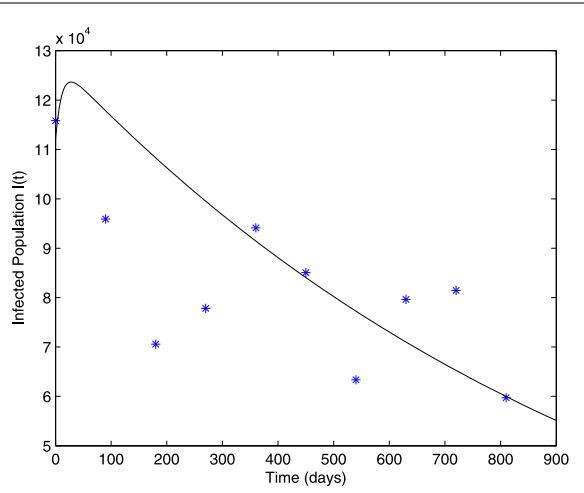

(b)

Figure 3 Figure 3 shows simulation of trends of infected individuals. Figure 3(a) shows simulations of model (3) at various initial values showing plot for people infected with rotavirus $/(t)$ when $R_{v}=0.4789$. Figure 3(b) shows real data from Table 2 fitted to model (3) with $R_{v}=0.9275$. 
Table 2 Number of children ( $<5$ years of age) who are infected with rotavirus in Kenya between January 2013 to July 2015

\begin{tabular}{lllll}
\hline Period & January-March & April-June & July-September & October-December \\
\hline 2013 & 116,672 & 96,619 & 71,097 & 78,389 \\
2014 & 94,796 & 85,681 & 63,805 & 80,212 \\
2015 & 82,035 & 60,159 & - & - \\
\hline
\end{tabular}

Source: data from Ministry of Health, Kenya Government.

immediate control measures be undertaken whenever there is an outbreak of a rotavirus infection. This will help in preventing a possible outbreak of an epidemic. This increase is also attributed to the fact that the rotavirus is a highly contagious virus that infects nearly all children by their fifth birthday. The figure also shows that the disease is persistent in the population; hence long-term control measures like vaccination, maintaining hygiene, and safe drinking water are recommended.

Figure 2 shows how varying $\epsilon$ affects both the susceptible and the vaccinated individuals. Figure 2(a) is plotted with $\epsilon=0.01$ and $R_{v}=1.6135$. In this figure, we observe that there are more susceptible individuals than vaccinated ones. Since $\epsilon$ is the expected decrease in the risk of infection as a result of vaccination, this variation may be a result of weak vaccines. The number of infections is likely to be high in this case because there are many susceptibles. When $\epsilon=0.001, R_{v}$ reduces to 1.2163 and the number of vaccinated individuals are now more than the susceptibles as evident in Figure 2(b), consequently resulting in few infectives. This therefore is an indication that stronger vaccines can significantly reduce rotavirus infections in a population. A very low value of $\epsilon$ indicates that the vaccine is very effective. It can therefore be concluded that vaccination at birth helps in reducing the number of infectives in a population. The effects of vaccination have been shown to be positive in areas where prevalence is high. These findings strongly support the search for and developments of effective vaccines by the WHO [9, 18, 36-38].

Figure 3(a), shows that, when $R_{v}=0.4789$, rotavirus infection can be wiped out of the population after a given period of time regardless of the number of individuals who were initially infected. This demonstrates that the fight against rotavirus infection can be successfully addressed if measures that can reduce $R_{v}$ are put in place. Measures like minimizing contacts between infected and susceptibles, and maintaining re-hydration of the infected are highly recommended. In Figure 3(b), we see that the control of the rotavirus infection is taking longer than in Figure 3(a). This is because $R_{v}=0.9275$ is high, compared to $R_{v}=0.4789$ of Figure 3(a). We have fitted real data from Table 2 to the model; see Figure 3(b). Though the fit appears not to be very accurate, it can still be used to predict the trend of the rotavirus infection in Kenya. The inaccuracy of the trend may be a result of the following.

- Lack of elaborate data because most hospitals in Kenya, especially in rural areas, started rotavirus vaccination in 2013. This is why our plot also started from the same year.

- Not all cases of rotavirus infections are reported to the hospitals, and also not all births are carried out in hospitals. This results in inaccurate reports on the state of rotavirus infections in the country.

- There is a delay in sending data from the sub-district hospitals to the Ministry headquarters. In the process, a lot of data and information get lost. 
- Most people in rural parts of Kenya are not following the vaccination schedules. This leads to false reports on the effectiveness of the vaccines.

- Finally, the model has mass-action transmission, which assumes homogeneous mixing. However, in reality, people do not mix homogeneously and that may result in disease clustering and persistence.

Though the model shows that the fight against rotavirus infection is promising in Kenya, a lot can still be achieved if the challenges noted above are addressed.

In summary, a mathematical model that shows the effects of vaccination on rotavirus infection has been developed and analyzed. The basic reproduction number $\left(R_{v}\right)$ has been established. It has been shown that an endemic equilibrium exists provided that $R_{v}>1$. Additionally, it has been proved that both the disease-free and the endemic equilibria are globally asymptotically stable. Numerical simulations have been performed using data attained from Ministry of Health, Kenya, to support the analysis and to show the effectiveness of vaccination as a way of controlling rotavirus infection. Real data has been fitted into the model to prove that it can be used to predict the rate of rotavirus infection in a population. From this work, it is recommended that all newborns be vaccinated (if possible) in order to effectively control rotavirus infection. For future work, we suggest that an investigation of how inclusion of seasonality will affect this model should be done. We also propose that future researchers should try and implement this model in a multi-group framework with heterogeneous contacts. For further details see the work by Wang and Cao [39].

Competing interests

The authors declare that they have no competing interests.

Authors' contributions

OLO performed the mathematical analysis of the model, and also wrote and typeset the manuscript. CW conducted the numerical simulations and discussions. XX proofread the final document. OGL formulated the model. All authors read and approved the final manuscript.

\section{Author details}

'Department of Mathematics, Harbin Institute of Technology, Harbin, 150001, P.R. China. ${ }^{2}$ Department of Mathematics, Egerton University, P.O. Box 536, Egerton, 20115, Kenya. ${ }^{3}$ Department of Mathematics, Masinde Muliro University of Science and Technology, P.O. Box 190, Kakamega, 50100, Kenya.

\section{Acknowledgements}

The authors of this work are very grateful to the Ministry of Health (Kenya) for allowing us to access and use their data. We are also very grateful to the Journal editors and the anonymous reviewers for their comments and recommendations, which have greatly improved our manuscript and make it more suitable for readers of the journal. Finally, we would like to appreciate the assistance given to us by the employees we dealt with at the Ministry.

Received: 27 September 2015 Accepted: 8 December 2015 Published online: 18 December 2015

References

1. White, LJ, Buttery, J, Cooper, B, Nokes, DJ, Medley, G: Rotavirus within day care centres in Oxfordshire, UK: characterization of partial immunity. J. R. Soc. Interface 5, 1481-1490 (2008)

2. Rotavirus Fact Sheet. Department of Health and Human Services, Centers for Disease Control and Prevention (2004)

3. Shim, E, Banks, H, Castillo-Chavez, C: Seasonality of rotavirus infection with its vaccination. Contemp. Math. 410, 327-347 (2006)

4. Anderson, EJ, Weber, SG: Rotavirus infection in adults. Lancet Infect. Dis. 4, 91-99 (2004)

5. Ruuska, T, Vesikari, T: Rotavirus disease in Finnish children: use of numerical scores for clinical severity of diarrhoeal episodes. Scand. J. Infect. Dis. 22, 259-267 (1990)

6. McNeal, MM, Bernstein, DI: Rotaviruses. In: Viral Infections of Humans, pp. 713-732. Springer, Berlin (2014)

7. Dennehy, PH: Transmission of rotavirus and other enteric pathogens in the home. Pediatr. Infect. Dis. J. 19(10), S103-S105 (2000)

8. Nitiema, LW, Nordgren, J, Ouermi, D, Dianou, D, Traore, AS, Svensson, L, Simpore, J: Burden of rotavirus and other enteropathogens among children with diarrhea in Burkina Faso. Int. J. Infect. Dis. 15(9), e646-e652 (2011)

9. Snelling, T, Markey, P, Carapetis, J, Andrews, R: Rotavirus infection in Northern Territory before and after vaccination. Microbiol. Aust. 33(2), 61-63 (2012) 
10. Wang, Y, Jin, Z, Yang, Z, Zhang, ZK, Zhou, T, Sun, GQ: Global analysis of an SIS model with an infective vector on complex networks. Nonlinear Anal., Real World Appl. 13(2), 543-557 (2012)

11. Wang, Y, Jin, Z: Global analysis of multiple routes of disease transmission on heterogeneous networks. Phys. A, Stat. Mech. Appl. 392(18), 3869-3880 (2013)

12. Hochwald, C, Kivela, L: Rotavirus vaccine, live, oral, tetravalent (RotaShield). Pediatr. Nursing 25(2), 203-204 (1998)

13. Jain, $P$, Jain, A: Waterborne viral gastroenteritis: an introduction to common agents. In: Water and Health, pp. 53-74. Springer, Berlin (2014)

14. Linhares, A, Gabbay, Y, Mascarenhas, J, Freitas, R, Flewett, T, Beards, G: Epidemiology of rotavirus subgroups and serotypes in Belem, Brazil: a three-year study. Ann. Inst. Pasteur Virol. 139 88-99 (1988)

15. Velázquez, FR, Matson, DO, Calva, JJ, Guerrero, ML, Morrow, AL, Carter-Campbell, S, Glass, RI, Estes, MK, Pickering, LK, Ruiz-Palacios, GM: Rotavirus infection in infants as protection against subsequent infections. N. Engl. J. Med. 335(14), 1022-1028 (1996)

16. Parashar, UD, Bresee, JS, Gentsch, JR, Glass, Rl: Rotavirus. Emerg. Infect. Dis. 4(4), 561-570 (1998)

17. Dennehy, PH: Rotavirus infection: a disease of the past? Infect. Dis. Clin. North Am. 29(4), 617-635 (2015)

18. Parez, N: Rotavirus gastroenteritis: why to back up the development of new vaccines? Comp. Immunol. Microbiol. Infect. Dis. 31(2), 253-269 (2008)

19. Parashar, UD, Hummelman, EG, Bresee, JS, Miller, MA, Glass, RI: Global illness and deaths caused by rotavirus disease in children. Emerg. Infect. Dis. 9(5), 565-572 (2003)

20. Reither, K, Ignatius, R, Weitzel, T, Seidu-Korkor, A, Anyidoho, L, Saad, E, Djie-Maletz, A, Ziniel, P, Amoo-Sakyi, F, Danikuu, F, Danour, S, Otchwemah, RN, Schreier, E, Bienzle, U, Stark, K, Mockenhaupt, FP: Acute childhood diarrhoea in northern Ghana: epidemiological, clinical and microbiological characteristics. BMC Infect. Dis. 7, 104 (2007)

21. Kiulia, NM, Nyaundi, JK, Peenze, I, Nyachieo, A, Musoke, RN, Steele, AD, Mwenda, JM: Rotavirus infections among HIV-infected children in Nairobi, Kenya. J. Trop. Pediatr. 55(5), 318-323 (2009)

22. Wardlaw, T, Salama, P, Brocklehurst, C, Chopra, M, Mason, E: Diarrhoea: why children are still dying and what can be done. Lancet 375(9718), 870-872 (2010)

23. Bishop, RF, Davidson, G, Holmes, I, Ruck, B: Virus particles in epithelial cells of duodenal mucosa from children with acute non-bacterial gastroenteritis. Lancet 302(7841), 1281-1283 (1973)

24. Heffernan, J, Smith, R, Wahl, L: Perspectives on the basic reproductive ratio. J. R. Soc. Interface 2(4), 281-293 (2005)

25. Diekmann, O, Heesterbeek, J, Metz, JA: On the definition and the computation of the basic reproduction ratio $R_{0}$ in models for infectious diseases in heterogeneous populations. J. Math. Biol. 28(4), 365-382 (1990)

26. van den Driessche, $P$, Watmough, J: Reproduction numbers and sub-threshold endemic equilibria for compartmental models of disease transmission. Math. Biosci. 180, 29-48 (2002)

27. Li, J, Blakeley, D, Smith, RJ: The failure of RO. Comput. Math. Methods Med. 2011, 527610 (2011)

28. Eigenwillig, A: Real root isolation for exact and approximate polynomials using Descartes' rule of signs (2010)

29. Stroud, KA, Booth, DJ: Engineering Mathematics. Palgrave Macmillan, Basingstoke (2013)

30. Salle, JL, Lefschetz, S: Stability by Liapunov's Direct Methods with Applications. Elsevier, Amsterdam (1961)

31. Wiggins, S: Introduction to Applied Nonlinear Dynamical Systems and Chaos, vol. 2, p. 110. Springer, Berlin (2003)

32. Vargas-De-León, C: On the global stability of SIS, SIR and SIRS epidemic models with standard incidence. Chaos Solitons Fractals 44(12), 1106-1110 (2011)

33. Agency, Cl: The CIA World Factbook 2012. Skyhorse Publishing, New York (2012)

34. Tate, JE, Rheingans, RD, O'Reilly, CE, Obonyo, B, Burton, DC, Tornheim, JA, Adazu, K, Jaron, P, Ochieng, B, Kerin, T, Calhoun, L, Hamel, M, Laserson, K, Breiman, RF, Feikin, DR, Mintz, ED, Widdowson, MA: Rotavirus disease burden and impact and cost-effectiveness of a rotavirus vaccination program in Kenya. J. Infect. Dis. 200, suppl. 1, S76-S84 (2009)

35. Vesikari, T, Matson, DO, Dennehy, P, Van Damme, P, Santosham, M, Rodriguez, Z, Dallas, MJ, Heyse, JF, Goveia, MG, Black, SB, et al.: Safety and efficacy of a pentavalent human-bovine (WC3) reassortant rotavirus vaccine. N. Engl. J. Med. 354, 23-33 (2006)

36. Vesikari, T, Isolauri, E, D'Hondt, E, Delem, A, André, F, Zissis, G: Protection of infants against rotavirus diarrhoea by RIT 4237 attenuated bovine rotavirus strain vaccine. Lancet 323(8384), 977-981 (1984)

37. Glass, Rl, Parashar, UD, Bresee, JS, Turcios, R, Fischer, TK, Widdowson, MA, Jiang, B, Gentsch, JR: Rotavirus vaccines: current prospects and future challenges. Lancet 368(9532), 323-332 (2006)

38. Flores, J, Gonzalez, M, Perez, M, Cunto, W, Perez-Schael, I, Garcia, D, Daoud, N, Chanock, R, Kapikian, A: Protection against severe rotavirus diarrhoea by rhesus rotavirus vaccine in Venezuelan infants. Lancet 329(8538), 882-884 (1987)

39. Wang, Y, Cao, J: Global dynamics of multi-group SEl animal disease models with indirect transmission. Chaos Solitons Fractals 69, 81-89 (2014) 